\title{
Os benefícios da laserterapia de baixa intensidade associados a exercícios domiciliares em idosos com osteoartrite de joelho
}

\author{
The benefits of low-intensity laser therapy associated with home \\ exercises in elderly knee osteoarthritis
}

\author{
Thaysson Silva Abreu', Edilma Marinho Garcia Sanches², Guilherme Marcelino Kuriki', \\ Johnny Massahiro Oshiro4, Fabio Chittero Boldrini' ${ }^{5}$, Márcia Midori Morimoto ${ }^{6}$
}

'Autor para correspondência. Universidade Municipal de São Caetano do Sul. São Caetano do Sul, São Paulo, Brasil. ORCID: 0000-0001-8612-1441. thayssonbuto@hotmail.com 2Universidade Municipal de São Caetano do Sul. São Caetano do Sul, São Paulo, Brasil. ORCID: 0000-0001-5225-8044. edilmamarinho19@gmail.com ${ }^{3}$ Universidade Municipal de São Caetano do Sul. São Caetano do Sul, São Paulo, Brasil. ORCID: 0000-0002-8518-8350. kuriki1998@gmail.com ${ }^{4}$ Universidade Municipal de São Caetano do Sul. São Caetano do Sul, São Paulo, Brasil. ORCID: 0000-0002-1728-5044. johnnyoshiro583@yahoo.com 5Universidade Municipal de São Caetano do Sul. São Caetano do Sul, São Paulo, Brasil. ORCID: 0000-0002-8307-8748. fcbfisio@gmail.com ${ }^{6}$ Universidade Municipal de São Caetano do Sul. São Caetano do Sul, São Paulo, Brasil. ORCID: 0000-0003-3449-729X.morimoto.marcia@prof.uscs.edu.br

\begin{abstract}
RESUMO | INTRODUÇÃO: A Osteoartrite (OA) é uma doença musculoesquelética com predomínio na população idosa. O tratamento com Laserterapia tem sido um dos métodos seguros e não invasivos para o tratamento da OA. OBJETIVO: Observar a influência na dor, rigidez e função em idosos antes e depois da aplicação da laserterapia associados a exercícios domiciliares. MÉTODOS: Dez pacientes idosos com OA de joelho, submetidos a 12 sessões de Laserterapia, 3 vezes por semana e orientados a realizarem exercícios domiciliares durante o período do estudo. Foi utilizado o Laser de baixa potência Class 3B Chattanooga, com energia total de 36J (divididos em 6 pontos - 6J por ponto) conforme a dosagem da World Association of Laser Therapy (WALT). Para mensuração do quadro álgico foi utilizados a Escala Visual Analógica (EVA); Índice de Osteoartrite de Western Ontario (WOMAC) e Índice de Lequesne para dor, rigidez e funcionalidade. Os exercícios domiciliares foram explicados e demonstrados pelo avaliador e orientado a realizar 3 vezes por semana durante 12 sessões. RESULTADOS: Observamos que após aplicações de Laserterapia e exercícios domiciliares, não houve melhora na EVA, porém com melhora significativa nos quesitos WOMAC geral $(p=0,01)$ e função $(p=0,001)$ enquanto os demais não houve significância $(p>0,5)$ quando avaliados por meio do t-test, já na comparação entre questionários houve correlação forte em WOMAC e LEQUESNE com a Correlação de Pearson. CONCLUSÃO: Pode-se concluir que a Laserterapia associada a exercícios domiciliares não evidencia melhora significativa do quadro álgico através da EVA, podendo ser uma resposta subjetiva com dificuldade de compreensão pelos pacientes, porém foi observado melhora significativa na evolução funcional do paciente medidas pelos questionários WOMAC e LEQUESNE nos pacientes com OA de joelho.
\end{abstract}

PALAVRAS-CHAVE: Osteoartrite. Terapia com luz de baixa intensidade. Artropatias. Dor. Atividade do dia a dia.
ABSTRACT | INTRODUCTION: Osteoarthritis (OA) is a musculoskeletal disease with predominance in the elderly population. Laser therapy has been one of the safe and non-invasive methods for treating OA. OBJECTIVE: To observe the influence on pain, stiffness and function in the elderly before and after the application of home exercise laser therapy. METHODS: Ten elderly patients with knee OA who underwent 12 laser therapy sessions 3 times a week. The Chattanooga Class 3B Low Power Laser was used, with a total energy of 36 (divided into 6 points - 6J per point) according to the dosage of the World Association of Laser Therapy (WALT). We used the Visual Analog Scale (VAS) for pain; Western Ontario Osteoarthritis Index (WOMAC) and Lequesne Index for pain, stiffness and functionality. Home exercises were explained and demonstrated by the evaluator and instructed to perform 3 times a week during 12 sessions. RESULTS: We observed that after laser therapy and home exercises, there was no improvement in VAS but there was significant improvement in the general WOMAC $(p=0.01)$ and function $(p=0.001)$, while the others were not significant $(p>0,5)$, when compared to the t-test, when compared to the Pearson correlation, there was an improvement in the WOMAC and LEQUESNE questionnaires. CONCLUSION: It can be concluded that after applying home exercise laser therapy, there was no significant improvement in pain through VAS, which may be a subjective response with difficulty for patients to understand, but there was a significant improvement in the patient's functional evolution measured by the questionnaires. WOMAC and LEQUESNE in patients with knee OA.

KEYWORDS: Osteoarthritis. Low-level light therapy. Joint diseases. Pain. Activities of daily living. 


\section{Introdução}

A Osteoartrite (OA) é tida como uma doença musculoesquelética predominante na população idosa, sendo assim um dos motivos mais comuns de limitação funcional e dependência e é classificada em primária ou idiopática, quando não é possível determinar sua causa, ou secundária, nos casos em que um ou mais fatores etiológicos podem ser apontados'. A articulação mais atingida é o joelho com causa desconhecida, sendo algumas causas etiológicas o fator sobrecarga, fatores ocupacionais e de sobrepeso, provocando dor articular protocinética, diminuição de força muscular e incapacidade funcional ${ }^{4}$. A OA de joelho é uma doença com sinais inflamatórios e processo degenerativo que provoca a destruição da cartilagem articular e leva a uma deformidade da articulação, essa doença, clinicamente, possui características de dor, crepitação, alargamento ósseo e limitação funcional progressiva, e assim podendo levar à deformidades articulares ${ }^{7}$.

Ainda não existe uma cura para a doença e o tratamento tem como objetivo o alivio da dor, melhora da função e da qualidade de vida em relação à saúde ${ }^{8}$. O tratamento recomendado deve seguir uma ordem: tratamento não farmacológico, terapia farmacológica e por último cirúrgico. O tratamento não farmacológico trás menos riscos e complicações, por isso deve ser mais focado e bastante utilizado antes de passar para a terapia farmacológica. Dentre os tratamentos não farmacológicos temos: acupuntura, terapia manual (massagem terapêutica, mobilização articular e manipulação), cinesioterapia (equilibrio corporal, coordenação motora, flexibilidade, resistência e força muscular de membros inferiores), termoterapia (calor superficial e frio), ultrassom, eletroestimulação elétrica transcutânea e laserterapia, que tem sido um dos métodos seguros e não invasivos que ultimamente tem atraído a atenção de muitos pesquisadores e especialistas para a intervenção na osteoartrite de joelho ${ }^{4,10}$.

A Laserterapia de baixa intensidade (LBI) é uma modalidade de tratamento não invasivo e de baixo custo, que por sua vez apresenta efeitos fisiológicos como: antiinflamatório, regeneração tecidual, modulador da atividade celular e analgésico, o efeito analgésico é o resultado da diminuição da inflamação por meio da reabsorção de exsudatos e da eliminação de substâncias algiogênicas ${ }^{11}$. A LBI é uma fonte de luz monocromática, tendo um efeito não térmico e estimula propriedades reparadoras da cartilagem em seres humanos ${ }^{9}$. Diversos resultados encontrados em uma série de estudos sugerem que o recurso promove um aumento da síntese de colágeno, aumento da proliferação e diferenciação de osteoblastos e fibroblastos, aumento da respiração mitocondrial e síntese de adenosina trifosfato (ATP), maior recrutamento de macrófagos e fibroblastos, aumento da angiogênese, aumento da atividade fagocitária, que resultará na aceleração do reparo de tecidos ${ }^{12}$. Além disso, os efeitos fotobiológicos da radiação do laser, de acordo com Andadre, Clark e Ferreira ${ }^{13}$, podem ser classificados como de curto e longo prazo. A diferença é que a curto prazo o efeito é visto logo após a aplicação da irradiação, já a longo prazo aparecem horas ou dias após a irradiação, e geralmente, abrangem nova biossíntese celular, principalmente na fase proliferativa da inflamação.

A laserterapia tem efeito analgésico, contudo os meios pelos quais isso acontece continuam incertos, alguns desses meios seriam o aumento da ATP mitocondrial e oxigenação mitocondrial, melhora de neurotransmissores, envolvidos na modulação da dor como a serotonina e ações anti-inflamatórias ${ }^{9}$.

De acordo com Raymundo et al. ${ }^{4}$ outro tratamento é a cinesioterapia, com um programa de treinamento que tem foco em melhorar a aptidão global geral, o equilíbrio corporal, a coordenação motora, a flexibilidade, a resistência e a força muscular dos membros inferiores, melhorando a função global e os sintomas de pacientes com osteoartrite. O guia de condutas do European League Against Rheumatism (EULAR) enfatiza a importância dos exercícios na redução da dor e meIhora da função, apesar de deixar claro que o regime ideal de exercícios para o tratamento da doença ainda não foi determinado'.

Contudo, sabe-se que terapias que envolvem o LLLT produzem melhora da resistência e da qualidade da cartilagem, acelerando o processo de reparação do tecido e consequentemente fornece uma melhora na qualidade de vida, sendo assim extremamente importante a inclusão do mesmo dentro de protocolos que tratam pacientes com OAJ. 
O objetivo deste trabalho é verificar a melhora da dor e a melhora da função em pacientes idosos com osteoartrite de joelho através do questionário funcional.

\section{Metodologia}

Este estudo se caracteriza como experimental e descritivo sendo os pacientes recrutados na Clínica de Fisioterapia da Universidade Municipal de São Caetano do Sul. Foram selecionados indivíduos com idade igual ou superior a 60 anos, ambos os sexos, com diagnostico de osteoartrite de joelho, não possuindo nenhum distúrbio neurológico que promovas alterações cognitivas, não possuindo qualquer tipo de implante metálico e/ou marcapasso, sem histórico de trombose venoso profunda nas extremidades inferiores ou processos neoplásicos, seguindo os critérios de inclusão e exclusão pré-estabelecidos; em seguida foi obtida a assinatura do Termo de Consentimento Livre e Esclarecido, o projeto foi aprovado pelo comitê de ética e pesquisa da Universidade Municipal de São Caetano do Sul (04388018.5.0000.5510).

Uma primeira avaliação foi aplicada para coleta de dados. Nesta avaliação foi coletado informações pessoais, constituído de anamnese, exame físico, escala visual analógica e a aplicação dos questionários WOMAC e LEQUESNE junto com o cálculo do IMC $\left(\mathrm{kg} / \mathrm{m}^{2}\right)$.

Os questionários WOMAC e LEQUESNE foram aplicados pelo pesquisador. Após a primeira avaliação, foi explicado os exercícios domiciliares e entregue uma cartilha aos pacientes que iniciaram o tratamento com LBI.

Os exercícios domiciliares realizados pelos pacientes são baseados no estudo de Youssef, Muaidi, Shanb ${ }^{14}$.
1. Alongamento para os músculos quadríceps, isquiotibiais, adutores e panturrilha por 30 segundos, relaxamento por 10 segundos e repetido 3 vezes; Tempo total de alongamento $\approx 5$ minutos.

2. Os exercícios de fortalecimento incluíram extensão do joelho, levantamento da perna esticada e exercício de ajuste do quadríceps. A contração será mantida por 6 segundos, seguida de relaxamento por 10 segundos e repetida 8 vezes / ajustada em cada exercício

Os pacientes foram orientados a realizarem três series de oito repetições e instruídos a praticar os mesmos em suas residências como um programa domiciliar.

As intervenções foram realizadas três vezes por semana, durante quatro semanas, contabilizando um total de 12 sessões. Anteriormente e após cada sessão o paciente comunicou seu quadro álgico através da escala visual analógica. No decorrer das sessões os pacientes receberam orientações domiciliares como conservação de energia e proteção articula, além da aplicação do LLLT no membro acometido, um Laser class 3B, com comprimento de onda de $850 \mathrm{~nm}$, com saída de pulso de $5 \mathrm{~mW} / \mathrm{cm}^{2}$, de 6 pontos por $\mathrm{cm}^{2}$ de 6 Joules por ponto, conforme a escala de dosagem da World Association of Laser Therapy (WALT), com o paciente em decúbito dorsal e a caneta posicionada na região anterior do joelho sobre a interarticular, sendo 3 pontos na região medial e 3 pontos na região lateral do joelho, com tempo total de aproximadamente 20 minutos de terapia (Figura 1). 


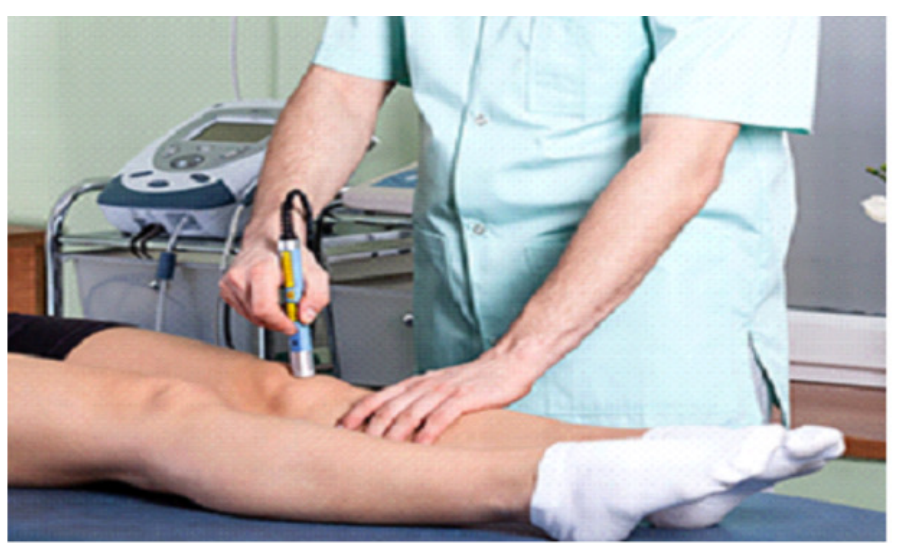

Ao final do tratamento, os pacientes foram submetidos a uma reavaliação fisioterapêutica, constituindo também de anamnese, exame físico, escala visual analógica e novamente pelos questionários de WOMAC e LEQUESNE.

A análise dos dados foi feita com o pacote estatístico GraphPad Prism. Todos os testes assumiram um nível de significância $a=5 \%$, utilizando o t-test e Correlação de Pearson para realização das comparações.

\section{Resultados}

Inicialmente pretendíamos recrutar 40 pacientes, no entanto, conseguimos entrar em contato com 24 pacientes, e desses apenas 13 compareceram para aplicação da avaliação inicial sendo selecionados 11 participantes, sendo 10 mulheres e 1 homem, conforme os critérios de inclusão e exclusão, com média de idade de 64,7 anos, índice de massa corporal (IMC) média de 27,74, apenas 1 participante foi excluído por não possuir $75 \%$ de presença, dentre os 10 participantes que sobraram somente 3 eram praticantes de alguma atividade física.

Os dados foram expostos em forma de média, evolução em percentual, correlação de Pearson e t-test (Tabela 1), com as variáveis EVA, WOMAC DOR, WOMAC RIGIDEZ, WOMAC CAPACIDADE FUNCIONAL, WOMAC GERAL e LEQUESNE.

Tabela 01. Valores em média, percentual de evolução, correlação em r-value e t-test em p-value das variáveis EVA, WOMAC e LEQUESNE (continua)

\begin{tabular}{|c|c|c|c|c|c|}
\hline & Inicial & Final & & & \\
\hline Variáveis & Média & Média & $\begin{array}{c}\text { Evolução em } \\
\text { percentual (\%) }\end{array}$ & $\begin{array}{c}\text { Correlação em r- } \\
\text { value }\end{array}$ & $\begin{array}{c}\text { T-test em p- } \\
\text { value }\end{array}$ \\
\hline EVA & 3,2 & 3,9 & $-21 \%$ & $r=0,60$ & $p>0,05$ \\
\hline WOMAC Geral & 41,6 & 33,7 & $19 \%$ & $r=0,94$ & $p=0,01$ \\
\hline
\end{tabular}


Tabela 01. Valores em média, percentual de evolução, correlação em r-value e t-test em p-value das variáveis EVA, WOMAC e LEQUESNE (conclusão)

\begin{tabular}{|c|c|c|c|c|c|}
\hline & Inicial & Final & & & \\
\hline Variáveis & Média & Média & $\begin{array}{l}\text { Evolução em } \\
\text { percentual (\%) }\end{array}$ & $\begin{array}{c}\text { Correlação em r- } \\
\text { value }\end{array}$ & $\begin{array}{l}\text { T-test em p- } \\
\text { value }\end{array}$ \\
\hline WOMAC dor & 7,7 & 6,7 & $13 \%$ & $r=0,72$ & $p>0,05$ \\
\hline WOMAC rigidez & 3,2 & 3,1 & $3 \%$ & $r=0,59$ & $p>0,05$ \\
\hline $\begin{array}{l}\text { WOMAC capacidade } \\
\text { funcional }\end{array}$ & 31,4 & 23,8 & $24 \%$ & $r=0,95$ & $p=0,001$ \\
\hline LEQUESNE & 9,5 & 7,9 & $17 \%$ & $r=0,75$ & $p>0,05$ \\
\hline
\end{tabular}

Os dados do presente estudo foram abordados em forma de gráficos e tabela conforme a seguir.

Os dados a seguir demostram as comparações entre a EVA inicial e final e WOMAC (Dor) inicial e final, WOMAC Fragmentado, WOMAC inicial e final x Lequesne inicial e final, WOMAC Geral inicial e final, Lequesne inicial e final, evolução em percentual do WOMAC.

O gráfico 1 demostra o quadro álgico apresentado pelos participantes do atual estudo, antes e depois da intervenção da Laserterapia (Gráfico 1), através da Escala Visual Analógica ( $r=0,60$ - correlação moderada) e WOMAC dor ( $r=0,72$ - correlação forte), obtendo-se desta forma diferença significativa em correlação de Pearson, porém não se obteve significância através do t-test $(p>0,05)$.

Gráfico 1. Média da comparação entre EVA inicial e final e WOMAC Dor inicial e final

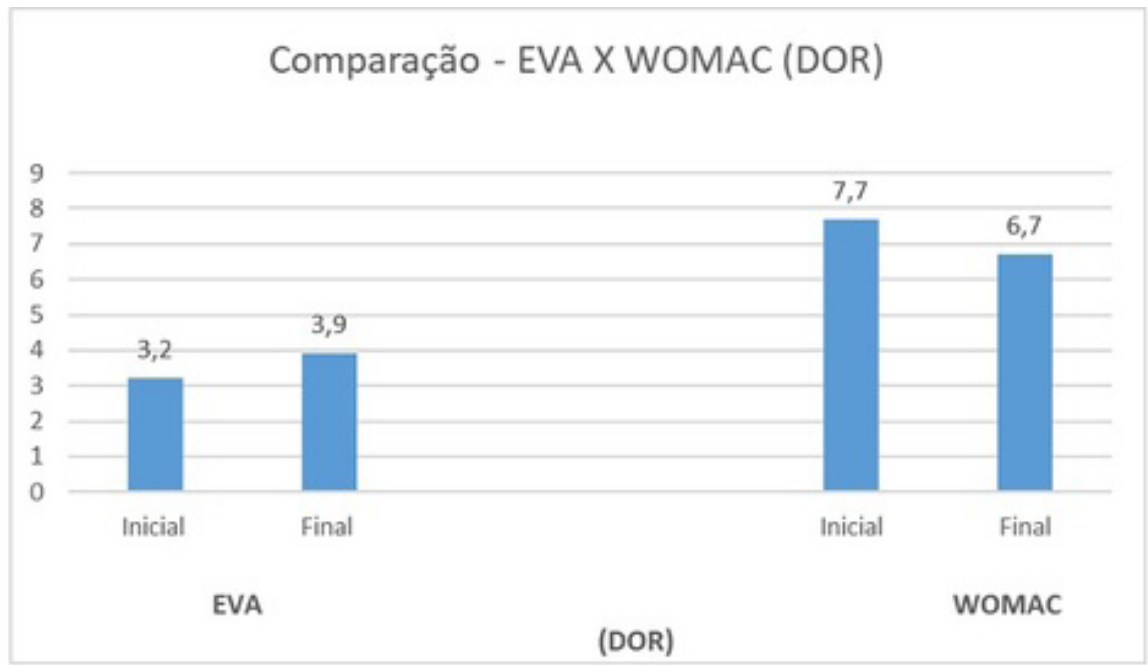


Na comparação do questionário WOMAC Geral inicial e final, foi analisado melhora após intervenção com uso da Laserterapia, apresentando diferença significativa ( $r=0,94$ - correlação muito forte), obtendo-se também melhora significativa em t-test $(p=0,01)$.

O gráfico 2 representa a média do WOMAC Fragmentado, sendo separado por dor inicial e final ( $r=0,72$ - correlação forte), rigidez inicial e final ( $r=0,59$ - correlação moderada) e capacidade funcional inicial e final ( $r=0,95$ - correlação muito forte), já não se obteve significância no quesito WOMAC dor e rigidez $(p>0,05)$ porém no WOMAC função apresentou significância em $t$-test $(0,001)$.

Gráfico 2. Média da comparação entre WOMAC Dor inicial e final, WOMAC Rigidez inicial e final e WOMAC Capacidade funcional inicial e final

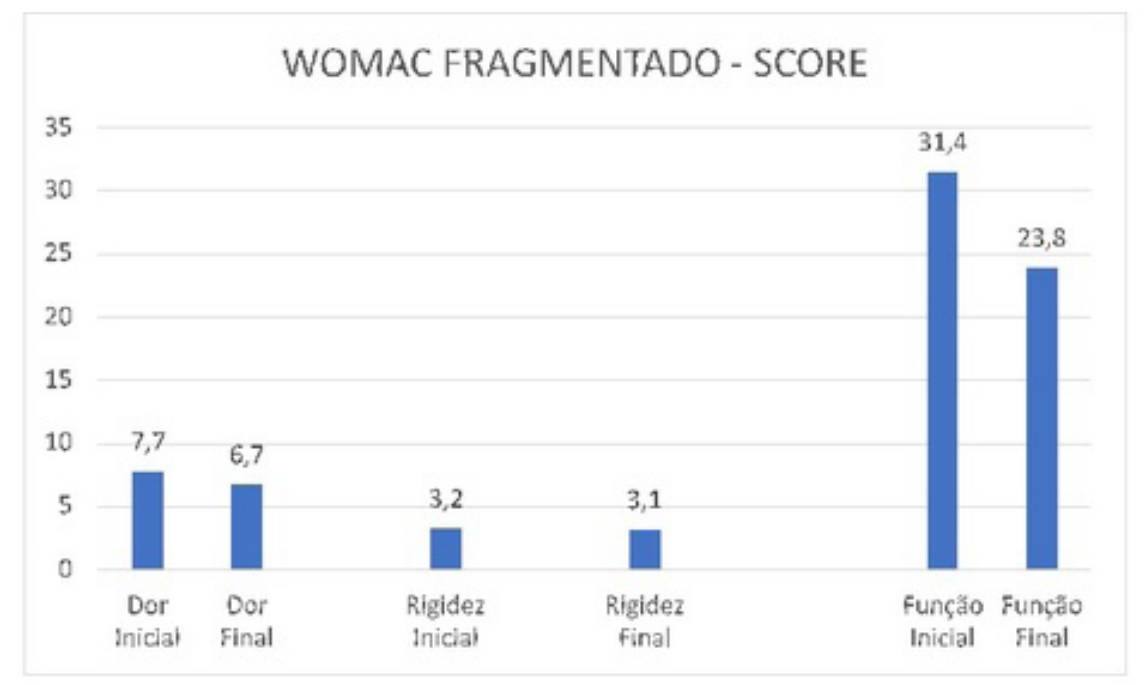

O gráfico 3 representa a média do WOMAC geral inicial e final ( $p=0,94$ - correlação muito forte) com Lequesne Geral inicial e final ( $p=0,75$ - correlação forte), onde demonstra que se obteve significância no WOMAC geral $(p=0,01)$ porém não se obteve significância em Lequesne geral $(p>0,05)$.

Gráfico 3. Média de comparação entre WOMAC Geral inicial e final e Lequesne Geral incial e final

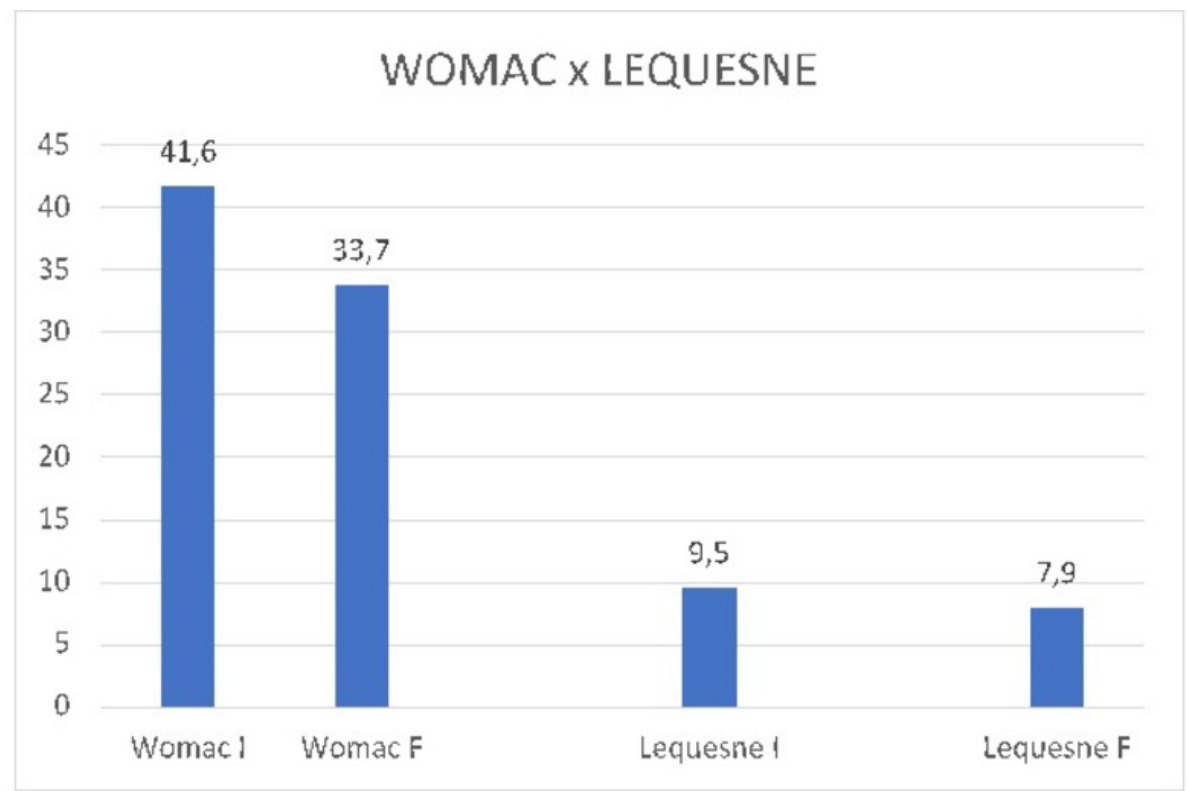


Na comparação do questionário algofuncinal de Lequesne inicial e final, foi analisado melhora após intervenção com uso da Laserterapia apresentando diferença significativa ( $r=0,75$ - correlação forte), porém não se obteve significância em t-test $(p>0,05)$.

\section{Discussão}

A Osteoartrite $(O A)$ é uma doença crônica degenerativa com predomínio da população idosa, sendo assim a causa mais comum de incapacidade, envolvendo dor e limitações nas atividades de vida diária ${ }^{13}$. A OA é determinada como uma deficiência da cartilagem articular por consequências de motivos mecânicos, genéticos, hormonais, ósseos e metabólicos, que causam um desgaste da cartilagem articular ${ }^{6}$.

Existem vários tratamentos para $\mathrm{OA}$, como o tratamento não farmacológico, terapia farmacológica e por último a cirurgia. Tratando-se do não farmacológico, esse é o que traz menos riscos e complicações, como por exemplo a Laserterapia, que tem sido um dos métodos mais seguros e não invasivos que tem atraído atenção de especialistas para a intervenção na OA de joelho'. Trazendo como benefícios a regeneração tecidual, melhora da síntese proteica e promoção da contenção da dor ${ }^{10}$.

Achados de alguns estudos evidenciam a efetividade da Laserterapia na funcionalidade (Alfredo et al. ${ }^{14}$; Alghadir et al. ${ }^{15}$ ) assim como no presente estudo.

No estudo realizado por Alfredo et al. ${ }^{14}$, foi realizado laserterapia durante 03 semanas e após a aplicação de laser foram realizados exercícios durante 08 semanas, como restulado obteve uma melhora significativa no WOMAC funcionalidade ( $p=0,002$ após laser e após exercícios) e WOMAC geral ( $p=0,008$ após laser e $p=0,003$ após exercícios), como no presente estudo $(p=0,001$ e $p=0,01$ respectivamente). O WOMAC dor também apresentou melhora significativa $(p=0,033$ após laser e $\mathrm{p}=0,001$ após exercícios), diferente do presente estudo $(p>0,05)$, que pode ter sido causado pelos exercícios (03 sessões por semana, durante 08 semanas e 45 minutos cada sessão).
Os dados encontrados no estudo de Fukuda et al. ${ }^{7}$, apresentou bons resultados na questão da EVA $(p<0,001)$ e no questionário de Lequesne $(p<0,001)$, diferente do presente estudo que não obteve diferença significativa nessas avaliações $(p>0,05)$. Estes resultados podem ser justificados pela quantidade de pacientes que se comprometeram no estudo, aumentando assim a probabilidade de possuir um resultado mais fidedigno.

O estudo de Alghadir et al. ${ }^{15}$, encontrou uma diferença significativa em todas as avaliações realizadas $(p<0,05)$, que foram: a EVA e o questionário WOMAC. O presente estudo não apresentou diferença significativa na EVA, WOMAC dor e WOMAC rigidez (todos $p>0,05$ ), podendo ser justificado pela quantidade de pacientes, como no estudo de Fukuda et al. ${ }^{7}$, ambos tiveram uma amostra maior comparado ao estudo apresentado.

Foi possível encontrar uma grande melhora da EVA $(p<0,001)$ no estudo de Rashoud et al. ${ }^{16}$, que por sua vez utilizaram uma dosagem menor que a recomendada pela WALT (5 pontos com 1,2) cada, e 40 segundos por ponto), diferente do presente estudo que utilizou os parâmetros recomendados pela WALT e não obteve diferença significativa na $\operatorname{EVA}(p>0,05)$. Pode ser justificado pela idade dos pacientes utilizados em cada estudo, a idade média do estudo de Rashoud et al. ${ }^{16}$, foi de 54 anos, e no presente estudo foi de 70 anos, podendo ter um aumento da dificuldade de compreensão da escala visual analógica e possuindo menor neuroplasticidade.

Os resultados encontrados nesse estudo não mostram um desfecho positivo no aspecto dor através da escala visual analógica (EVA) e pode ser justificada pela dificuldade de compreensão dos participantes durante a avaliação desta variável, porém pode-se observar melhora significativa tanto no WOMAC geral e WOMAC capacidade funcional. Desta forma, entende-se necessário mais estudos com o tema em questão e amostra superior, porém é possível afirmar que por se tratar de questionários validados na literatura, fidedignos e englobarem quesitos como Dor, Função e Rigidez, a Laserterapia associada aos exercícios domiciliares pode ser considerada uma abordagem viável e positiva para pacientes com osteoartrite de joelho. 


\section{Conclusão}

Pode-se concluir que após aplicação da Laserterapia associado aos exercícios domiciliares, não houve meIhora significativa do quadro álgico através da EVA, porém no quesito WOMAC geral e WOMAC capacidade funcional foi identificado melhora significativa, além da evolução funcional através dos questionários WOMAC e LEQUESNE nos pacientes com OA de joelho.

\section{Agradecimentos}

A Profa Rosamaria Rodrigues Garcia por sempre nos ajudar tirando dúvidas e nos auxiliando no decorrer do trabalho; ao Profo Mario Caxambu Neto por disponibilizar a clínica de Fisioterapia da Universidade de São Caetano para a realização do trabalho; a Lucimar Moreira Simões por estar sempre com a gente durante a realização de todo trabalho.

\section{Contribuições individuais}

Abreu TS participou da concepção, delineamento, busca, análise estatística, coleta de dados e avaliação do paciente, aplicação da laserterapia no paciente. Boldrini F participou da concepção, delineamento, análise estatística e na orientação do trabalho. Kuriki GM e Sanches EMG participaram da concepção, delineamento, busca, coleta de dados, avaliação e reavaliação do paciente. Oshiro JM participou da concepção, delineamento, busca, explicação do programa domiciliar aos pacientes, aplicação da laserterapia no paciente e análise estatística.

\section{Conflitos de interesses}

Nenhum conflito financeiro, legal ou político envolvendo terceiros (governo, empresas e fundações privadas, etc.) foi declarado para nenhum aspecto do trabalho submetido (incluindo, mas não se limitando a subvenções e financiamentos, participação em conselho consultivo, desenho de estudo, preparação de manuscrito, análise estatística, etc.).

\section{Referências}

1. Alfredo PP. A eficácia de baixa intensidade associada a exercícios em pacientes com osteoartrose de joelho: estudo randomizado e duplo-cego [tese]. São Paulo: Faculdade de Medicina da Universidade de São Paulo; 2011.
2. Santos Junior EC, Borges AMM, Bianco BAV, Andrade RF. Perfil epidemiológico dos pacientes com osteoartrose de joelho atendidos no serviço de fisioterapia reumatológica em duas clinicas escola de Maceió. In: Anais do $4^{\circ}$ Congresso Internacional de Envelhecimento Humano; 2015; Maceió; 2015.

3. Raymundo SF, Caldas Junior ACT, Maiworm A, Cader SA. Comparação de dois tratamentos fisioterapêuticos na redução da dor e aumento da autonomia funcional de idoso com gonartrose. Rev Bras Geriatr Gerontol. 2014;17(1):129-140. doi: $10.1590 /$ $\underline{\mathrm{S} 1809-98232014000100013}$

4. Melo SIL, Oliveira J, Detânico RC, Palhano R, Schwinden RM, Andrade MC et al. Avaliação da força muscular de flexores e extensores de joelho em indivíduos com e sem osteoartrose. Revista Bras de Cineantropom Desempenho Hum. 2008;10(4):355340. doi: 10.5007/1980-0037.2008v10n4p335

5. Garrido CA, Sampaio TCFVS, Ferreira FS. Estudo comparativo entre a classificação radiológica e análise macro e microscópia das lesões na osteoartrose do joelho. Rev Bras Ortop. 2011;46(2):155-159. doi: 10.1590/S0102-36162011000200007

6. Camanho GL, Imamura M, Arendt-Nielsen L. Gênese da dor na artrose. Rev Bras Ortop. 2011;46(1):14-17. doi: 10.1590/S0102$\underline{36162011000100002}$

7. Fukuda VO, Fukuda TY, Guimarães M, Shiwa S, Lima BDC, Martins RABL et al. Eficácia a curto prazo do laser de baixa intensidade em pacientes com osteoartrite do joelho: ensaio clínico aleatório, placebo-controlado e duplo cego. Rev Bras Ortop. 2011;46(5):526-533. doi: 10.1590/S010236162011000500008

8. Rayegani SM, Raeissadat SA, Heidari S, Moradi-Joo M. Safety and Effectiveness of Low-Level Laser Therapy in Patients With Knee Osteoarthritis: a systematic review and meta-analysis. J Lasers Med Sci. 2017;8(supl 1):12-19. doi: 10.15171/jlms.2017.s3

9. Andrade TNC, Frare JC. Estudo comparativo entre os efeitos de técnicas de terapia manual isoladas e associadas à laserterapia de baixa potência sobre a dor em pacientes com disfunção temporomandibular. RGO. 2008;56(3):287-295.

10. Oliveira P. Os efeitos do laser terapêutico em modelo experimental de osteoartrite em ratos [tese]. São Carlos: Universidade Federal de São Calos; 2013.

11. Andrade FSSD, Clark RMO, Ferreira ML. Efeitos da laserterapia de baixa potência na cicatrização de feridas cutâneas. Rev Col Bras Cir. 2014;41(2):129-133. doi: 10.1590/S0100$\underline{69912014000200010}$ 
12. Youssef EF, Muaidi QI, Shanb AA. Effect of lase therapy on chronic osteoarthritis of the knee in older subjects. J Lasers Med Sci. 2019;7(2):112-119. doi: 10.15171/jlms.2016.19

13. Fernandes L, Hagen KB, Bijlsma JW, Andreassen $\mathrm{O}$, Christensen $\mathrm{P}$, Conaghan PG et al. EULAR recommendations for the non-pharmacological core management of hip and knee osteoarthritis. Ann Rheum Dis. 2013;72(7):1125-1135. doi: 10.1136/annrheumdis-2012-202745

14. Alfredo PP, Bjordal JM, Dreyer SH, Meneses SR, Zaguetti $\mathrm{G}$, Ovanessian $\mathrm{V}$ et al. Efficacy of low level laser therapy associated with exercises in knee osteoarthritis: a randomized double-blind study. Clin Rehabil. 2011;26(6):523-533. doi: 10.1177/0269215511425962

15. Alghadir A, Omar MT, Al-Askar AB, Al-Muteri NK. Effect of lowlevel laser therapy in patients with chronic knee osteoarthritis: a single-blinded randomized clinical study. Lasers Med Sci. 2014;29(2):749-755. doi: 10.1007/s10103-013-1393-3

16. Al Rashoud AS, Abboud RJ, Wang W, Wigderowitz C. Efficacy of low-level therapy applied at acupuncture points in knee osteoarthritis: a randomized double-blind comparative trial. Physiotherapy. 2014;100(3):242-248. doi: 10.1016/j. physio.2013.09.007 